\title{
Tafamidis, a Noninvasive Therapy for Delaying Transthyretin Familial Amyloid Polyneuropathy: Systematic Review and Meta-Analysis
}

\author{
Yinan Zhao ${ }^{\mathrm{a} *}$ \\ Yanguo Xin $^{\text {b* }}$ \\ Zhuyin Song ${ }^{c}$ \\ Zhiyi $\mathrm{He}^{\mathrm{a}}$ \\ Wenyu $\mathrm{Hu}^{\mathrm{b}}$ \\ aDepartments of Neurology and \\ ${ }^{\mathrm{b}}$ Cardiology, The First Affiliated Hospital \\ of China Medical University, \\ Shenyang, China \\ 'Department of Neurology, \\ The People's Hospital \\ of Liaoning Province, \\ Shenyang, China
}

Background and Purpose Tafamidis functions to delay the loss of function in transthyretin familial amyloid polyneuropathy (TTR-FAP), which is a rare inherited amyloidosis with progressive sensorimotor and autonomic polyneuropathy. This systematic literature review and meta-analysis evaluated the efficacy and safety of tafamidis in TTR-FAP patients, with the aim of improving the evidence-based medical evidence of this treatment option for TTP-FAP.

Methods A systematic search of the English-language literature in five databases was performed through to May 31, 2018 by two reviewers who independently extracted data and assessed the risk of bias. We extracted efficacy and safety outcomes and performed a meta-analysis. Statistical tests were performed to check for heterogeneity and publication bias.

Results The meta-analysis identified six relevant studies. The tafamidis group showed smaller changes from baseline in the Neuropathy Impairment Score-Lower Limbs [mean difference $(\mathrm{MD})=-3.01,95 \%$ confidence interval $(\mathrm{CI})=-3.26$ to $-2.75, p<0.001]$ and the Norfolk Quality of Life-Diabetic Neuropathy total quality of life score (MD=-6.67, $95 \% \mathrm{CI}=-9.70$ to $-3.64, p<0.001)$, and a higher modified body mass index ( $\mathrm{MD}=72.45,95 \% \mathrm{CI}=69.41$ to $75.49, p<0.001)$, with no significant difference in total adverse events [odds ratio $(\mathrm{OR})=0.69,95 \% \mathrm{CI}=0.35$ to $1.35, p=$ 0.27]. The incidence of adverse events did not differ between tafamidis and placebo treatment except for fatigue $(\mathrm{OR}=0.13,95 \% \mathrm{CI}=0.02$ to $0.72, p=0.02)$ and hypesthesia $(\mathrm{OR}=0.16,95 \%$ $\mathrm{CI}=0.03$ to $0.92, p=0.04$ ).

Conclusions This systematic review and meta-analysis has demonstrated that tafamidis delays neurologic progression and preserves a better nutritional status and the quality of life. The rates of adverse events did not differ between the patients in the tafamidis and placebo groups. Tafamidis might be a safer noninvasive option for patients with TTR-FAP.

Key Words amyloid neuropathies, familial, meta-analysis, safety, therapeutics.
Received June 20, 2018

Revised September 23, 2018

Accepted September 28, 2018

\section{Correspondence}

Wenyu $\mathrm{Hu}, \mathrm{PhD}$

Department of Cardiology,

The First Affiliated Hospital

of China Medical University,

No.155, Heping

Shenyang 110001, China

Tel +86-024-83283166

Fax +86-024-83283166

E-mail huwen0320@sohu.com

*These authors contributed equally to this work.

\section{INTRODUCTION}

Transthyretin familial amyloid polyneuropathy (TTR-FAP) is a rare inherited amyloidosis disorder associated with mutation in the TTR gene that affects more than 38,000 patients worldwide and manifests as a progressive sensorimotor and autonomic polyneuropathy. ${ }^{1-3}$ The gene mutations lead to destabilization of the tetrameric structure of the TTR protein, such as dissociation, misfolding, and aggregation. The TTR protein usually acts as an amyloid target to the peripheral nerve tissue, and axonal degeneration initiating from small myelinated and unmyelinated nerve fibers to larger myelinated fibers, causing sensory syndromes including pain and temperature sensation in the lower extremities, motor impairment, muscle weakness, progressive and autonomic gastrointestinal, urogenital, and cardiovascular

(®) This is an Open Access article distributed under the terms of the Creative Commons Attribution Non-Commercial License (https://creativecommons.org/licenses/by-nc/4.0) which permits unrestricted non-commercial use, distribution, and reproduction in any medium, provided the original work is properly cited. 
dysfunctions, or even death within a decade from onset.,

TTR is a plasma protein consisting of 127 -amino-acid monomers, which is mainly secreted by the liver and functions as a backup transporter for thyroxine and a primary transporter for the retinol-binding protein/vitamin A complex. ${ }^{6}$ The TTR gene is located on chromosome 18q12.1 in human. More than 120 described point mutations have been identified related to TTR-FAP and degeneration.-10 The most-common TTR-FAP cases are linked with the replacement of valine by methionine at position 30 of the TTR protein (V30M). ${ }^{7,11,12}$

Liver transplantation has been considered as the primary option for patients with TTR-FAP, which replaces the main source of the variant TTR protein with a normal donor organ to halt the progression of neuropathy in well-selected patients with mild hereditary TTR-FAP, and achieves an acceptable 20 -year survival rate of more than $50 \%$ despite multiple disadvantages. ${ }^{13,14}$ However, as an invasive treatment, liver transplantation is also accompanied by high perioperative mortality and morbidity even when chronic immunosuppressive treatment is applied. ${ }^{15,16}$ Additionally, in spite of a higher survival rate for early-onset V30M TTR-FAP patients, poor responses in late-onset V30M TTR-FAP and non-V30M TTRgene-mutant patients have been reported. ${ }^{14,17}$

Given this limitation, a noninvasive therapy with superior medication safety and curative effect is urgently needed.

Tafamidis is an orally administered amyloid fibrils inhibitor that can potently occupy thyroxine-binding sites and stabilize tetramers with high specificity, and is the first approved disease-modifying medicine for treating TTR-FAP. ${ }^{18}$ Many recent researches have focused on the efficacy and safety of tafamidis in TTR-FAP patients from diverse populations and regions. Tafamidis is emerging as a first-line medicine option to replace liver transplantation. ${ }^{19-22}$

The present study is the first systematic literature review and meta-analysis performed to evaluate the efficacy and safety of tafamidis in TTR-FAP patients, with the aim of improving the medical-evidence base for applying it as a treatment option for TTP-FAP.

\section{METHODS}

\section{Search strategy}

This systematic literature review and meta-analysis were performed using the methodology suggested by the Preferred Reporting Items for Systematic Reviews and Meta-Analysis (PRISMA) guidelines. A systematic search of the Englishlanguage literature was performed through to May 31, 2018 in the following databases: MEDLINE, PubMed, Embase, Web of Science, and Cochrane Library. The following keywords used were: "tafamidis," "transthyretin familial amyloid poly- neuropathy," “TTR-FAP," "V30MTTR,” “nonV30MTTR," "randomized," and "randomly." These keywords were combined with appropriate Boolean operators, and further relevant articles were searched for by also checking the reference lists of all of the identified publications. After completing the literature searches, the titles and abstracts of the study reports were screened by Z.Y.N. and X.Y.G., and any disagreement was resolved by discussion with or (if necessary) adjudication by H.W.Y.

\section{Selection criteria}

The following inclusion criteria were applied in the present systematic review and meta-analysis: 1) a randomized, placebo-controlled trial (RCT) with at least 12 months of follow-up, 2) patients diagnosed with TTR-FAP, and 3) studies that compared the effects of $20 \mathrm{mg}$ of tafamidis self-administered once daily as a 1:1 meglumine salt or matching placebo. Studies with the following characteristics were considered ineligible: 1) studies that did not include outcomes information, 2) case reports or studies for which insufficient information was provided about the patients, and 3) studies without sufficient data to allow the incidence of mortality or other outcomes to be determined.

\section{Endpoints}

\section{Primary endpoints}

Primary efficacy analyses were performed to assess the efficacy of tafamidis, including changes from baseline in the Neuropathy Impairment Score-Lower Limbs (NIS-LL) and the Norfolk Quality of Life-Diabetic Neuropathy total quality of life (TQOL) score. The NIS-LL quantifies the motor and reflex functions in the lower limbs to assessing the disease severity in TTR-FAP. ${ }^{23,24}$ The Norfolk Quality of Life scale utilizes a patient-reported questionnaire that provides a TQOL score ranging from -2 (best) to 138 (worst). ${ }^{25}$ The efficacy endpoints were obtained before the drug regimen was changed.

\section{Secondary endpoints}

The modified body mass index ( $\mathrm{mBMI}$ ), including the change therein, was used to assess the efficacy of tafamidis. $\mathrm{mBMI}$ is calculated as the product of the BMI and the serum albumin concentration, and measures wasting and autonomic gastrointestinal function.

\section{Study selection, data extraction, and quality assessment}

The following basic information was extracted for each study independently by Z.Y.N. and S.Z.Y. using a standard form: surname of the first author, year of publication, country in 
which the procedure was performed, study design, group assignment, number of patients and their basic characteristics, follow-up duration, and outcomes (Table 1).

The modified Jadad quality scoring scale evaluates various parameters such as the generation of random sequences, distribution methods, randomized concealment, and whether a blind method was adopted. ${ }^{26}$ The total Jadad score ranges from 0 to $7 ; 1-3$ points for a low-quality study and $4-7$ points for a high-quality study. Two raters performed the quality assessment independently (Z.Y.N. and X.Y.G.), with disagreements resolved by seeking the opinion of the third rater (H.W.Y.) (Table 2). Publication bias was evaluated using Egger's regression test, and Begg's adjusted rank correlation test (Table 3).

\section{Statistical analysis}

The statistical analysis was performed independently by X.Y.G. and H.W.Y. according to recommendations from the PRISMA statement and the Cochrane handbook from the Cochrane Collaboration. The pooled mean difference (MD) or odds ratio (OR) with 95\% confidence intervals (CIs) were calculated for each principal dichotomous variable outcome. Cochrane's Q-test and I-squared $\left(\mathrm{I}^{2}\right)$ were used to evaluate the statistical heterogeneity for the pooled results. The combined result was regarded as having heterogeneity if $p<0.05$ or $\mathrm{I}^{2}>50 \%$. A fixed-effect model was used to conduct a metaanalysis when the data showed inconspicuous heterogeneity, otherwise a random-effect model was applied. Subgroup analysis was also conducted in our meta-analysis.

The meta-analysis was performed using RevMan software (version 5.3, Cochrane Collaboration; Copenhagen, Den- mark). The presence of publication bias was assessed with Egger's regression test and Begg's adjusted rank correlation test using the meta bias commands in STATA (version 14.0, StataCorp, College Station, TX, USA). All of the statistical tests were two-sided, and $p<0.05$ was considered statistically significant.

\section{RESULTS}

\section{Literature selection}

The database searches yielded 110 entries, of which 76 were excluded due to duplications, reviews, or irrelevance. Of the 34 publications that qualified for abstract review, 17 were dropped for the following reasons: only a single treatment arm ( $n=3)$, comparison of the effect of tafamidis on TTRFAP between different TTR gene mutations $(n=2)$, follow-up duration of less than 1 year $(n=1)$, lack of clarity of the NIS-LL or Norfolk Quality of Life score $(n=3)$, treatment regimens in the control group not in accordance with our inclusion criteria $(n=8)$. Full-text articles were assessed for the remaining 17 publications, and 11 were excluded with the following reasons: sample too small $(n=8)$ or insufficient data $(n=3)$. We therefore finally enrolled six trials for further analysis. The PRISMA flow diagram for study selection is shown in Fig. 1.

\section{Included trials and bias assessment}

The characteristics of the included patients are listed in detail in Table $1 .{ }^{27-32}$ The results for publication bias in the six comparative trials are presented in Table 3.

Table 1. Baseline characteristics of the included studies

\begin{tabular}{|c|c|c|c|c|c|c|c|c|}
\hline \multirow{2}{*}{ Reference (year) } & \multicolumn{2}{|c|}{ Sample size, males } & \multicolumn{2}{|c|}{ Age, years } & \multicolumn{2}{|c|}{ Symptom duration, months } & \multirow{2}{*}{$\begin{array}{l}\text { Clinical } \\
\text { stage }\end{array}$} & \multirow{2}{*}{$\begin{array}{c}\text { Follow-up } \\
\text { period, months }\end{array}$} \\
\hline & Tafamidis & Placebo & Tafamidis & Placebo & Tafamidis & Placebo & & \\
\hline Barroso et al., $2017^{31}$ & $38(18)$ & $37(16)$ & $40.7 \pm 14.1$ & $38.6 \pm 13.8$ & $45.2 \pm 55.3$ & $38.6 \pm 34.6$ & Early & 72 \\
\hline Coelho et al., $2013^{30}$ & $38(17)$ & $33(15)$ & $44.3 \pm 15.0$ & $33.0 \pm 14.6$ & $94.8 \pm 84.1$ & $61.4 \pm 40.7$ & Not reported & 30 \\
\hline Suhr et al., $2014^{29}$ & $38(17)$ & $33(15)$ & $43.0 \pm 14.6$ & $40.5 \pm 14.4$ & $76.5 \pm 83.7$ & $42.8 \pm 40.4$ & Not reported & 30 \\
\hline Keohane et al., $2017^{27}$ & 48 & 44 & - & - & - & - & Early & 18 \\
\hline Gundapaneni et al., $2018^{28}$ & $64(30)$ & $61(30)$ & $39.8 \pm 12.7$ & $38.4 \pm 12.9$ & $47.0 \pm 48.4$ & $34.7 \pm 32.9$ & Not reported & 12 \\
\hline Coelho et al., $2012^{32}$ & $64(32)$ & $61(26)$ & $39.8 \pm 12.7$ & $38.4 \pm 12.9$ & $47.0 \pm 48.4$ & $34.7 \pm 32.9$ & Early & 18 \\
\hline
\end{tabular}

Data are mean $\pm S D$ values.

Table 2. Jadad scores for the included studies

\begin{tabular}{|c|c|c|c|c|c|}
\hline Reference (year) & Randomization & Concealment of allocation & Double blinding & Withdrawals and dropouts & Jadad score \\
\hline Barroso et al., $2017^{31}$ & 2 & 1 & 2 & 1 & 6 \\
\hline Coelho et al., $2013^{30}$ & 2 & 1 & 2 & 1 & 6 \\
\hline Suhr et al., $2014^{29}$ & 2 & 1 & 2 & 1 & 6 \\
\hline Keohane et al., $2017^{27}$ & 2 & 1 & 2 & 1 & 6 \\
\hline Gundapaneni et al., $2018^{28}$ & 2 & 1 & 2 & 1 & 6 \\
\hline Coelho et al., $2012^{32}$ & 2 & 1 & 2 & 1 & 6 \\
\hline
\end{tabular}


Table 3. Results from the meta-analysis of various outcomes and publication bias

\begin{tabular}{|c|c|c|c|c|c|c|c|c|c|}
\hline & \multirow{2}{*}{ MD/OR } & \multirow{2}{*}{$95 \% \mathrm{Cl}$} & \multirow{2}{*}{$p$} & \multirow{2}{*}{ Model } & \multicolumn{2}{|c|}{ Heterogeneity } & \multicolumn{2}{|c|}{$p$ value for publication bias } & \multirow{2}{*}{ Studies (n) } \\
\hline & & & & & $p$ & $I^{2}(\%)$ & Begg's test & Egger's test & \\
\hline NIS-LL LS mean change & -3.01 & $-3.26,-2.75$ & $<0.001$ & Fixed & 0.37 & 0 & 0.296 & 0.077 & 3 \\
\hline mBMI mean change & 72.45 & $69.41,75.49$ & $<0.001$ & Fixed & 0.88 & 0 & 0.734 & 0.130 & 4 \\
\hline TQOL mean change & -6.67 & $-9.70,-3.64$ & $<0.001$ & Random & $<0.001$ & 88 & 0.296 & 0.486 & 3 \\
\hline Mortality & 0.97 & $0.24,3.98$ & 0.97 & Fixed & 0.46 & 0 & 1.000 & - & 2 \\
\hline Subjects with $\mathrm{AE}$ & 0.46 & $0.18,1.17$ & 0.10 & Fixed & 0.19 & 39 & 0.296 & 0.194 & 3 \\
\hline Serious AEs & 0.88 & $0.38,2.05$ & 0.77 & Fixed & 0.53 & 0 & 1.000 & - & 2 \\
\hline AEs leading to drug discontinuation & 1.22 & $0.32,4.71$ & 0.77 & Fixed & 0.86 & 0 & 1.000 & - & 2 \\
\hline Diarrhea & 1.48 & $0.74,2.99$ & 0.27 & Fixed & 0.87 & 0 & 0.296 & 0.063 & 3 \\
\hline Urinary tract infection & 1.11 & $0.60,2.07$ & 0.74 & Fixed & 0.20 & 38 & 1.000 & 0.022 & 3 \\
\hline Pain in extremity & 1.13 & $0.29,4.42$ & 0.87 & Random & 0.17 & 47 & 1.000 & - & 2 \\
\hline Influenza & 0.59 & $0.29,1.17$ & 0.13 & Fixed & 0.20 & 37 & 0.296 & 0.032 & 3 \\
\hline Headache & 0.66 & $0.33,1.32$ & 0.24 & Fixed & 0.49 & 0 & 1.000 & 0.635 & 3 \\
\hline Nasopharyngitis & 1.35 & $0.65,2.81$ & 0.42 & Fixed & 0.86 & 0 & 0.296 & 0.057 & 3 \\
\hline Nausea & 1.21 & $0.48,3.08$ & 0.69 & Fixed & 0.37 & 0 & 1.000 & - & 2 \\
\hline Vomiting & 0.78 & $0.32,1.89$ & 0.58 & Fixed & 0.83 & 0 & 1.000 & - & 2 \\
\hline Punctate keratitis & 1.29 & $0.43,3.85$ & 0.65 & Fixed & 0.60 & 0 & 1.000 & - & 2 \\
\hline Back pain & 2.03 & $0.67,6.14$ & 0.21 & Fixed & 0.25 & 23 & 1.000 & - & 2 \\
\hline Vaginal infection & 2.37 & $0.59,9.47$ & 0.22 & Fixed & 0.50 & 0 & 1.000 & - & 2 \\
\hline Peripheral edema & 0.92 & $0.15,5.66$ & 0.92 & Random & 0.15 & 52 & 1.000 & - & 2 \\
\hline Constipation & 0.74 & $0.26,2.06$ & 0.56 & Fixed & 0.36 & 0 & 1.000 & - & 2 \\
\hline Upper respiratory tract infection & 0.96 & $0.37,2.49$ & 0.93 & Fixed & 0.82 & 0 & 0.296 & 0.075 & 3 \\
\hline Thermal burn & 0.88 & $0.40,1.95$ & 0.75 & Fixed & 0.97 & 0 & 1.000 & 0.928 & 3 \\
\hline Anxiety & 0.55 & $0.07,4.09$ & 0.55 & Random & 0.13 & 57 & 1.000 & - & 2 \\
\hline Depression & 2.46 & $0.69,8.76$ & 0.16 & Fixed & 0.22 & 32 & 1.000 & - & 2 \\
\hline Paresthesia & 0.34 & $0.12,1.00$ & 0.05 & Fixed & 0.44 & 0 & 1.000 & - & 2 \\
\hline Dizziness & 0.97 & $0.27,3.44$ & 0.96 & Fixed & 0.20 & 39 & 1.000 & - & 2 \\
\hline Hypesthesia & 0.16 & $0.03,0.92$ & 0.04 & Fixed & 0.64 & 0 & 1.000 & - & 2 \\
\hline Fatigue & 0.13 & $0.02,0.72$ & 0.02 & Fixed & 0.51 & 0 & 1.000 & - & 2 \\
\hline
\end{tabular}

AE: adverse event, Cl: confidence interval, LS mean: least-squares mean, mBMI: modified body mass index, MD: mean difference, NIS-LL: Neuropathy Impairment Score-Lower Limbs, OR: odds ratio, TQOL: total quality of life.

\section{Efficacy endpoints}

\section{Co-primary endpoints}

The NIS-LL and the least-squares (LS) mean changes in the NIS-LL from the baseline to the endpoint levels were reported for three of the included studies..$^{27,28,31}$ The overall metaanalysis revealed a statistically significant difference, with an MD of -3.01 in the LS mean change between the tafamidis and placebo groups, with no significant heterogeneity $(95 \% \mathrm{CI}=$ -3.26 to $-2.75, p<0.001, \mathrm{I}^{2}=0 \%$ ) (Fig. 2, Table 3, Supplementary Fig. 1 in the online-only Data Supplement).

The LS mean change from the baseline in the Norfolk Quality of Life-Diabetic Neuropathy TQOL score to the endpoint level was reported for three of the included studies. ${ }^{27,31,32}$ The overall meta-analysis revealed a statistically significant difference, with an MD of -6.67 in the LS mean change between the tafamidis and placebo groups, with a significant hetero- geneity ( $95 \% \mathrm{CI}=-9.70$ to $-3.64, p<0.001, \mathrm{I}^{2}=88 \%$ ) (Fig. 2 , Table 3, Supplementary Fig. 1 in the online-only Data Supplement).

\section{Secondary endpoint}

The change in mBMI was taken as the secondary endpoint for evaluating the nutritional status in the tafamidis and placebo groups, and four studies were included..$^{27,29,31,32}$ The overall meta-analysis revealed a statistically significant difference, with an MD of 72.45 for the mBMI change between the tafamidis and placebo groups ( $95 \% \mathrm{CI}=69.41$ to 75.49 , $p<0.001, \mathrm{I}^{2}=0 \%$ ) (Fig. 2, Table 3, Supplementary Fig. 1 in the online-only Data Supplement).

\section{Safety endpoints}

Adverse events were reported in detail for three studies. ${ }^{30-32}$ The proportion of patients reporting adverse events did not 


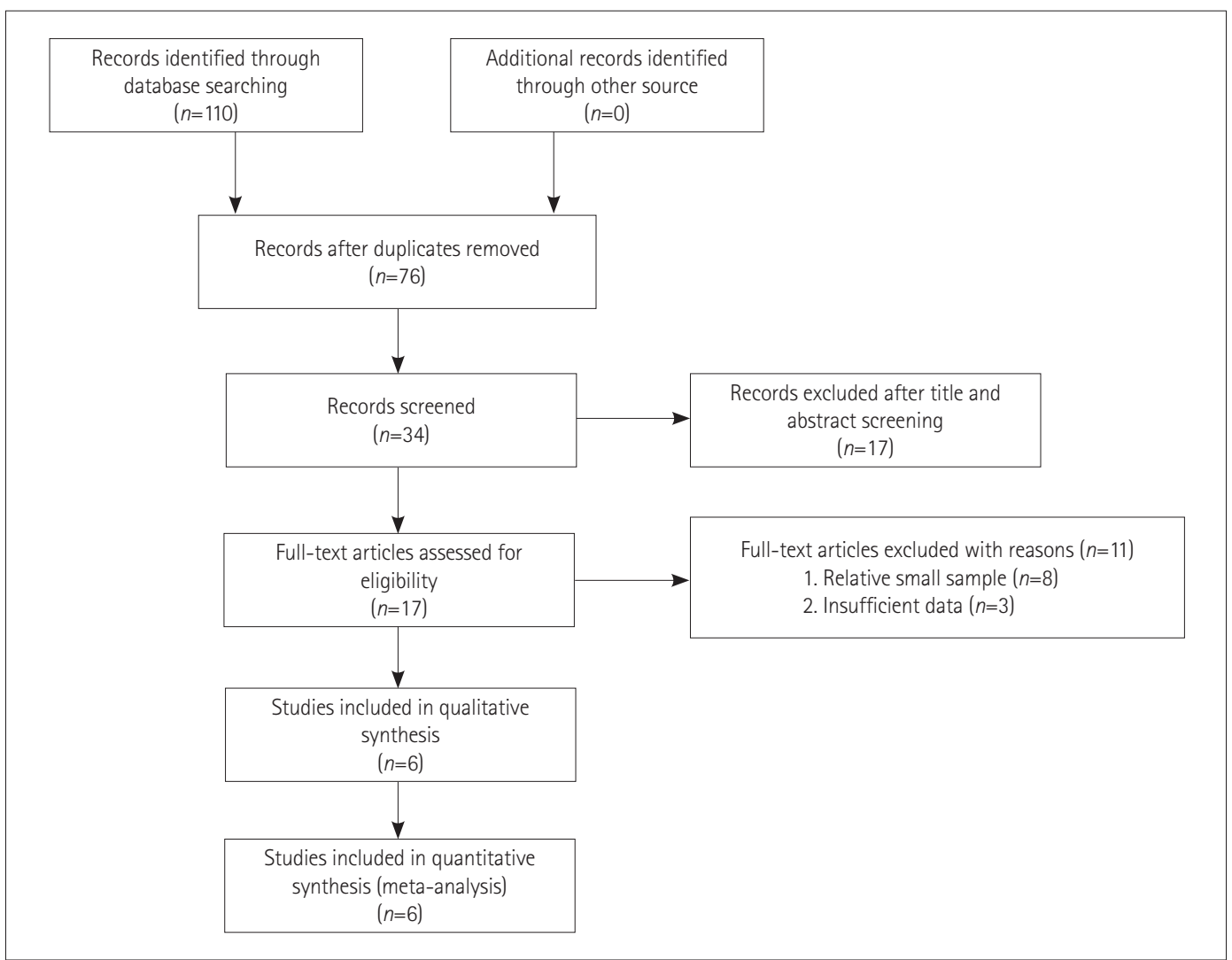

Fig. 1. Trial selection process.

differ between the groups: $132(89.8 \%)$ of the 147 patients in the tafamidis group versus 134 (95\%) of the 141 patients in the placebo group $\left(\mathrm{OR}=0.46,95 \% \mathrm{CI}=0.18\right.$ to $1.17, p=0.10, \mathrm{I}^{2}=$ 39\%) (Fig. 2, Table 3, Supplementary Fig. 2 in the online-only Data Supplement). Mortality events were reported for two of the studies. ${ }^{31,32}$ The proportion of such events did not differ between the groups (OR=0.97, 95\% $\mathrm{CI}=0.24$ to 3.98, $p=$ $0.97, \mathrm{I}^{2}=0 \%$ ) (Fig. 2, Table 3, Supplementary Fig. 2 in the online-only Data Supplement).

Subgroup analyses showed that most-common adverse events did not differ between the tafamidis and placebo groups (Fig. 2, Table 3, Supplementary Fig. 2 in the online-only Data Supplement). However, the proportion of patients reporting fatigue differed significantly between the two groups: 1 (0.01\%) of the 103 patients in the tafamidis group versus 10 (10\%) of the 100 patients in the placebo group $(\mathrm{OR}=0.13,95 \% \mathrm{CI}=0.02$ to $0.72, p=0.02, \mathrm{I}^{2}=0 \%$ ) (Fig. 2, Table 3, Supplementary Fig. 2 in the online-only Data Supplement). The proportion of patients reporting hypesthesia also differed significantly between the groups: $1(0.01 \%)$ of the 103 patients in the tafamidis group versus 8 ( $8 \%$ ) of the 100 patients in the placebo group ( $\mathrm{OR}=0.16,95 \% \mathrm{CI}=0.03$ to $0.92, p=0.04, \mathrm{I}^{2}=0 \%$ ) (Fig. 2, Table 3, Supplementary Fig. 2 in the online-only Data Supplement).
Quality and publication bias of the included studies The quality of the included studies was quantitatively assessed using the Jadad scale. All six included studies employed randomization, concealment of allocation, and double blinding, and all of them had a Jadad score of 6 points, indicating that they were of high quality (Table 2). ${ }^{27-32}$ Publication bias was tested using Egger's regression test and Begg's adjusted rank correlation test, and we found no significant correlation between effect size or other evidence of publication bias (Table 3).

\section{DISCUSSION}

We collected six double-blind RCTs that included patients with TTR-FAP to evaluate the efficacy and safety of tafamidis. The pooled results demonstrated the following: 1) tafamidis treatment achieved a significantly better result than placebo treatment in terms of the LS mean changes from the baseline in the NIS-LL, TQOL score, and mBMI, and 2) the incidence rates of adverse events were similar in the tafamidis and placebo treatment groups. Although subgroup analysis revealed that the most-common adverse events did not differ between the tafamidis and placebo groups, the risk of hypesthesia and fatigue were significantly lower in the tafamidis group than 


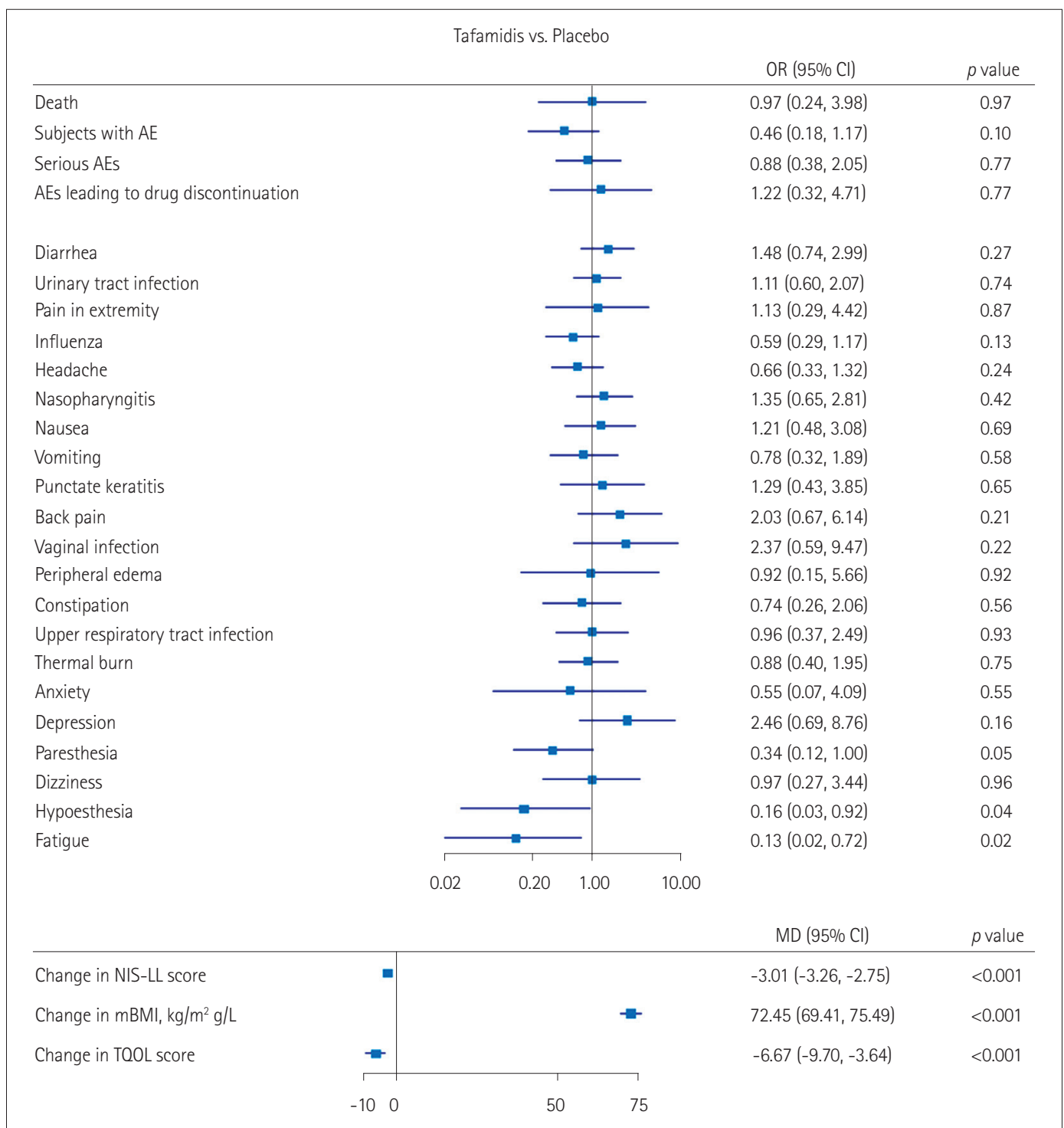

Fig. 2. Efficacy and safety endpoints. AE: adverse event, $\mathrm{Cl}$ : confidence interval, mBMI: modified body mass index, MD: mean difference, NIS-LL: Neuropathy Impairment Score-Lower Limbs, OR: odds ratio, TQOL: total quality of life.

in the placebo treatment group of patients with TTR-FAP.

A previous study found that tafamidis treatment did not affect patients with TTR amyloid cardiomyopathy. ${ }^{33}$ However, a recent multicenter, international, double-blind, placebocontrolled, phase 3 trial involving 441 patients with TTR amyloid cardiomyopathy randomly assigned to a tafamidis or placebo group for 30 months found that tafamidis was associated with reductions in all-cause mortality, cardiovascular-related hospitalizations, and the declines in functional capacity and quality of life as compared with placebo. ${ }^{34}$ This represents strong evidence that tafamidis is an effective therapy for patients with TTR amyloid cardiomyopathy.

In addition to clinical studies of the effect of tafamidis on TTR-FAP patients, several trials have examined the roles of a kinetic stabilizer (diflunisal) on TTR-FAP disease progres- sion. ${ }^{35-37}$ Diflunisal is a generic nonsteroidal anti-inflammatory drug that has been successfully complexed to the thyroxine binding site, and it has been demonstrated to kinetically stabilize circulating TTR protein tetramers, thereby inhibiting the release of the TTR protein monomer necessary for amyloidogenesis. ${ }^{38,39}$ These findings represent strong clinical evidence that kinetically stabilizing an amyloidogenic precursor protein (transthyretin) exerts beneficial effects on amyloid-related neurologic disease progression.

TTR gene silencing therapies that inhibit the hepatic production of the mutant and nonmutant TTR proteins via posttranscriptional gene silencing have recently received considerable attention in explorations of invasive treatments. Antisense oligonucleotides are synthetic strings of nucleotides designed to prevent the expression of a targeted protein by selectively 
binding to the RNA that encodes the targeted protein and thereby prevent translation. It was reported very recently that inotersen, a 2'-O-methoxyethyl-modified antisense oligonucleotide that inhibits hepatic production of TTR, improves the course of neurologic disease and the quality of life in patients with hereditary TTR amyloidosis. The limitations of this clinical trial included that it had insufficient statistical power for measuring the effects of inotersen treatment on cardiomyopathy. ${ }^{40}$ ISIS-TTR $\mathrm{RX}_{\mathrm{RX}}$ is a second-generation antisense inhibitor of the molecular target TTR, and is designed to bind within the nontranslated portion of the human TTR gene messenger RNA (mRNA) and thereby degrade this mRNA to prevent the production of both nonmutant and mutant TTR proteins. ${ }^{41}$

RNA interference (RNAi) therapy has also been used to suppress the production of TTR. ${ }^{42}$ RNAi is an endogenous cellular mechanism for controlling gene expression in which small interfering RNAs that are bound to the RNA-induced silencing complex mediate the cleavage of target mRNA. ${ }^{43,44} \mathrm{RNAi}$ based drugs harness endogenous posttranscriptional gene silencing pathways for therapeutic purposes. Two RNAi drugs (ALN-TTR02 and ALN-TT ${ }_{\text {Rsc }}$ ) have been developed that target the disease-causing mutant TTR gene mRNA in the liver for treating TTR-FAP. A greater efficacy in targeting the disease-causing mutant combined with lower risk and better toleration are expected for RNAi-based drugs targeting TTR-FAP. Patisiran is another investigational RNAi therapeutic agent, which specifically inhibits the hepatic synthesis of TTR. A recent randomized, placebo-controlled, phase 3 trial involving patients with hereditary TTR amyloidosis with polyneuropathy found that patisiran improved multiple clinical manifestations of hereditary TTR amyloidosis. ${ }^{45}$ That trial also yielded evidence that patisiran improved the cardiac manifestations of hereditary transthyretin amyloidosis. We also anticipate that RNAi-based drugs will be widely used for other hereditary diseases in the future. However, no previous clinical trial has compared the efficacy and safety between tafamidis and RNAi drugs for TTR-FAP patients. We therefore also look forward to such clinical studies, which could yield strong evidence of useful treatment options for TTR-FAP patients.

While the present study included six high-quality RCTs, it was also subject to some limitations. Firstly, because this meta-analysis focused on a rare disease, the statistical power was unavoidable restricted by the relative smallness of the sample of existing studies. Secondly, most of the included studies had both a prospective and open-label design, resulting in missing data and a possibility of bias. Thirdly, the inherent limitations of a meta-analysis such as publication bias cannot be ignored. Fourthly, the statistical heterogeneity was quantified for the outcome of TQOL mean changes. The heterogeneity of indicators of continuous variables is generally quite difficult to investigate. Due to a very small number of reports on TQOL mean changes and the different sample sizes and selection criteria applied in the studies, we were unable to conduct a subgroup analysis. Moreover, our sensitivity analysis (Supplementary Fig. 3 in the online-only Data Supplement) failed to identify the real sources of the heterogeneity. We speculate that the heterogeneity was caused by multiple factors such as age, sex, disease course, and treatment time.

In conclusion, this systematic review and meta-analysis of six RCTs has demonstrated that tafamidis exhibits a slower neurologic disease progression and better preservation of nutritional status and quality of life.

The rate of adverse events did not differ between the patients in the tafamidis and placebo groups. These findings indicate that tafamidis might be a safer noninvasive option for patients with TTR-FAP.

\section{Supplementary Materials}

The online-only Data Supplement is available with this article at https://doi.org/10.3988/jcn.2019.15.1.108.

\section{Conflicts of Interest}

The authors have no financial conflicts of interest.

\section{Acknowledgements}

The authors gratefully acknowledge financial support from China Scholarship Council.

\section{REFERENCES}

1. Schmidt HH, Waddington-Cruz M, Botteman MF, Carter JA, Chopra AS, Hopps M, et al. Estimating the global prevalence of transthyretin familial amyloid polyneuropathy. Muscle Nerve 2018;57:829-837.

2. Hund E, Linke RP, Willig F, Grau A. Transthyretin-associated neuropathic amyloidosis. Pathogenesis and treatment. Neurology 2001;56: 431-435.

3. Benson MD. The hereditary amyloidoses. Best Pract Res Clin Rheumatol 2003;17:909-927.

4. Andersson R. Familial amyloidosis with polyneuropathy. A clinical study based on patients living in northern Sweden. Acta Med Scand Suppl 1976;590:1-64.

5. Planté-Bordeneuve V, Lalu T, Misrahi M, Reilly MM, Adams D, Lacroix $\mathrm{C}$, et al. Genotypic-phenotypic variations in a series of 65 patients with familial amyloid polyneuropathy. Neurology 1998;51:708-714.

6. Monaco HL, Rizzi M, Coda A. Structure of a complex of two plasma proteins: transthyretin and retinol-binding protein. Science 1995;268: 1039-1041.

7. Connors LH, Lim A, Prokaeva T, Roskens VA, Costello CE. Tabulation of human transthyretin (TTR) variants, 2003. Amyloid 2003;10: 160-184.

8. Gertz MA, Benson MD, Dyck PJ, Grogan M, Coelho T, Cruz M, et al. Diagnosis, prognosis, and therapy of transthyretin amyloidosis. $J$ Am Coll Cardiol 2015;66:2451-2466.

9. Almeida MR, Alves IL, Terazaki H, Ando Y, Saraiva MJ. Comparative studies of two transthyretin variants with protective effects on familial amyloidotic polyneuropathy: TTR R104H and TTR T119M. Biochem 
Biophys Res Commun 2000;270:1024-1028.

10. Koike H, Misu K, Sugiura M, Iijima M, Mori K, Yamamoto M, et al. Pathology of early- vs late-onset TTR Met30 familial amyloid polyneuropathy. Neurology 2004;63:129-138.

11. Planté-Bordeneuve V, Said G. Familial amyloid polyneuropathy. Lancet Neurol 2011;10:1086-1097.

12. Saraiva MJ. Transthyretin mutations in health and disease. Hum Mutat 1995;5:191-196.

13. Sekijima Y, Kelly JW, Ikeda S. Pathogenesis of and therapeutic strategies to ameliorate the transthyretin amyloidoses. Curr Pharm Des 2008; 14:3219-3230.

14. Ericzon BG, Wilczek HE, Larsson M, Wijayatunga P, Stangou A, Pena JR, et al. Liver transplantation for hereditary transthyretin amyloidosis: after 20 years still the best therapeutic alternative? Transplantation 2015;99:1847-1854

15. Okamoto S, Wixner J, Obayashi K, Ando Y, Ericzon BG, Friman S, et al. Liver transplantation for familial amyloidotic polyneuropathy: impact on Swedish patients' survival. Liver Transpl 2009;15:1229-1235.

16. Winkler M, Brinkmann C, Jost U, Oldhafer K, Ringe B, Pichlmayr R. Long-term side effects of cyclosporine-based immunosuppression in patients after liver transplantation. Transplant Proc 1994;26:2679-2682.

17. Suhr OB, Larsson M, Ericzon BG, Wilczek HE; FAPWTR's investigators. Survival after transplantation in patients with mutations other than Val30Met: extracts from the FAP world transplant registry. Transplantation 2016;100:373-381.

18. Bulawa CE, Connelly S, Devit M, Wang L, Weigel C, Fleming JA, et al. Tafamidis, a potent and selective transthyretin kinetic stabilizer that inhibits the amyloid cascade. Proc Natl Acad Sci U S A 2012;109:96299634.

19. Plante-Bordeneuve V. Update in the diagnosis and management of transthyretin familial amyloid polyneuropathy. J Neurol 2014;261: 1227-1233.

20. Adams D, Cauquil C, Labeyrie C, Beaudonnet G, Algalarrondo V, Théaudin M. TTR kinetic stabilizers and TTR gene silencing: a new era in therapy for familial amyloidotic polyneuropathies. Expert Opin Pharmacother 2016;17:791-802.

21. Tong C, Blanco M, Goddard WA 3rd, Seinfeld JH. Thermodynamic properties of multifunctional oxygenates in atmospheric aerosols from quantum mechanics and molecular dynamics: dicarboxylic acids. Environ Sci Technol 2004;38:3941-3949.

22. Waddington Cruz M, Benson MD. A review of tafamidis for the treatment of transthyretin-related amyloidosis. Neurol Ther 2015;4: 61-79.

23. Kim DH, Zeldenrust SR, Low PA, Dyck PJ. Quantitative sensation and autonomic test abnormalities in transthyretin amyloidosis polyneuropathy. Muscle Nerve 2009;40:363-370.

24. Kolb NA, Smith AG, Singleton JR, Beck SL, Stoddard GJ, Brown S, et al. The association of chemotherapy-induced peripheral neuropathy symptoms and the risk of falling. JAMA Neurol 2016;73:860-866.

25. Vinik EJ, Hayes RP, Oglesby A, Bastyr E, Barlow P, Ford-Molvik SL, et al. The development and validation of the Norfolk QOL-DN, a new measure of patients' perception of the effects of diabetes and diabetic neuropathy. Diabetes Technol Ther 2005;7:497-508.

26. Jadad AR, Moore RA, Carroll D, Jenkinson C, Reynolds DJ, Gavaghan DJ, et al. Assessing the quality of reports of randomized clinical trials: is blinding necessary? Control Clin Trials 1996;17:1-12.

27. Keohane D, Schwartz J, Gundapaneni B, Stewart M, Amass L. Tafamidis delays disease progression in patients with early stage transthyretin familial amyloid polyneuropathy: additional supportive analyses from the pivotal trial. Amyloid 2017;24:30-36.

28. Gundapaneni BK, Sultan MB, Keohane DJ, Schwartz JH. Tafamidis delays neurological progression comparably across Val30Met and non-Val30Met genotypes in transthyretin familial amyloid polyneuropathy. Eur J Neurol 2018;25:464-468.

29. Suhr OB, Conceição IM, Karayal ON, Mandel FS, Huertas PE, Ericzon BG. Post hoc analysis of nutritional status in patients with transthyretin familial amyloid polyneuropathy: impact of tafamidis. Neurol Ther 2014;3:101-112.

30. Coelho T, Maia LF, da Silva AM, Cruz MW, Planté-Bordeneuve V, Suhr OB, et al. Long-term effects of tafamidis for the treatment of transthyretin familial amyloid polyneuropathy. J Neurol 2013;260:2802-2814.

31. Barroso FA, Judge DP, Ebede B, Li H, Stewart M, Amass L, et al. Long-term safety and efficacy of tafamidis for the treatment of hereditary transthyretin amyloid polyneuropathy: results up to 6 years. Amyloid 2017;24:194-204.

32. Coelho T, Maia LF, Martins da Silva A, Waddington Cruz M, PlantéBordeneuve V, Lozeron P, et al. Tafamidis for transthyretin familial amyloid polyneuropathy: a randomized, controlled trial. Neurology 2012;79:785-792.

33. Maurer MS, Grogan DR, Judge DP, Mundayat R, Packman J, Lombardo I, et al. Tafamidis in transthyretin amyloid cardiomyopathy: effects on transthyretin stabilization and clinical outcomes. Circ Heart Fail 2015;8:519-526.

34. Maurer MS, Schwartz JH, Gundapaneni B, Elliott PM, Merlini G, Waddington-Cruz M, et al. Tafamidis treatment for patients with transthyretin amyloid cardiomyopathy. N Engl J Med 2018;379:1007-1016.

35. Berk JL, Suhr OB, Obici L, Sekijima Y, Zeldenrust SR, Yamashita T, et al. Repurposing diflunisal for familial amyloid polyneuropathy: a randomized clinical trial. JAMA 2013;310:2658-2667.

36. Takahashi R, Ono K, Shibata S, Nakamura K, Komatsu J, Ikeda Y, et al. Efficacy of diflunisal on autonomic dysfunction of late-onset familial amyloid polyneuropathy (TTR Val30Met) in a Japanese endemic area. J Neurol Sci 2014;345:231-235.

37. Sekijima Y, Tojo K, Morita H, Koyama J, Ikeda S. Safety and efficacy of long-term diflunisal administration in hereditary transthyretin (ATTR) amyloidosis. Amyloid 2015;22:79-83.

38. Sekijima Y, Dendle MA, Kelly JW. Orally administered diflunisal stabilizes transthyretin against dissociation required for amyloidogenesis. Amyloid 2006;13:236-249.

39. Miller SR, Sekijima Y, Kelly JW. Native state stabilization by NSAIDs inhibits transthyretin amyloidogenesis from the most common familial disease variants. Lab Invest 2004;84:545-552.

40. Benson MD, Waddington-Cruz M, Berk JL, Polydefkis M, Dyck PJ, Wang AK, et al. Inotersen treatment for patients with hereditary transthyretin amyloidosis. N Engl J Med 2018;379:22-31.

41. Benson MD, Kluve-Beckerman B, Zeldenrust SR, Siesky AM, Bodenmiller DM, Showalter AD, et al. Targeted suppression of an amyloidogenic transthyretin with antisense oligonucleotides. Muscle Nerve 2006;33:609-618.

42. Coelho T, Adams D, Silva A, Lozeron P, Hawkins PN, Mant T, et al. Safety and efficacy of RNAi therapy for transthyretin amyloidosis. $N$ Engl J Med 2013;369:819-829.

43. Fire A, Xu S, Montgomery MK, Kostas SA, Driver SE, Mello CC. Potent and specific genetic interference by double-stranded RNA in Caenorhabditis elegans. Nature 1998;391:806-811.

44. Elbashir SM, Harborth J, Lendeckel W, Yalcin A, Weber K, Tuschl T. Duplexes of 21-nucleotide RNAs mediate RNA interference in cultured mammalian cells. Nature 2001;411:494-498.

45. Adams D, Gonzalez-Duarte A, O’Riordan WD, Yang CC, Ueda M, Kristen AV, et al. Patisiran, an RNAi therapeutic, for hereditary transthyretin amyloidosis. N Engl J Med 2018;379:11-21. 